\title{
El potencial de colonización micorrícico-arbuscular varía entre prácticas agrícolas y sitios en diferentes áreas geográficas de la Región Pampeana
}

\author{
M. Noelia Cofré ${ }^{1, \mathbb{D}}$; Carlos Urcelay ${ }^{1}$; Luis G. Wall ${ }^{2}$; Laura DomíngueZ ${ }^{1} \&$ \\ Alejandra BecerRa
}
${ }^{1}$ Instituto Multidisciplinario de Biología Vegetal (IMBIV), CONICET, Universidad Nacional de Córdoba. Córdoba, Argentina. ${ }^{2}$ Laboratorio de Bioquímica, Microbiología e Interacciones Biológicas en Suelo (LBMIBS), Departamento de Ciencia y Tecnología, Universidad Nacional de Quilmes. Bernal, Argentina.

\begin{abstract}
Resumen. Para evaluar las comunidades fúngicas es necesario identificar las especies presentes, cuantificar la densidad de propágulos y también determinar su infectividad. En este estudio se evaluó, mediante un ensayo experimental, el potencial de colonización micorrícico-arbuscular (PCM) de tres suelos bajo diferentes sistemas de cultivo (pastizal natural, rotación de cultivo y monocultivo de soja en siembra directa), en cuatro sitios ubicados en diferentes áreas geográficas de la Región Pampeana (Bengolea, Monte Buey, Pergamino y Viale), y se usó a Vicia villosa Roth como planta testigo. Los usos del suelo y los sitios tuvieron efecto sobre el PCM. Como esquema de rotación, el monocultivo mostró el mayor potencial para colonizar las raíces de Vicia villosa con respecto al pastizal natural, y la rotación de cultivo en la mayoría de las combinaciones planteadas, a diferencia de lo encontrado antes para los mismos suelos, donde el monocultivo disminuyó la riqueza de las comunidades micorrícicas determinadas por la densidad y la diversidad de esporas. Estos resultados remarcan las limitaciones de los paradigmas de interpretación de los sistemas simbióticos generados a partir de ensayos en condiciones controladas y el gran desconocimiento que aún existe sobre el funcionamiento de las interacciones planta-microorganismo en el suelo. Alternativamente, estos resultados podrían ser consecuencia de un efecto inhibitorio de la mayor fertilidad N-P en los suelos con rotación de cultivo respecto de los suelos bajo monocultivo debido a un manejo de reposición histórica de nutrientes por fertilización, ausente en los casos de monocultivo. El uso de una única especie vegetal en los ensayos de infectividad también podría estar sesgando los resultados, ya que limitaría, por especificidad de hospedador, su expresión en la totalidad de la comunidad micorrícica del suelo.
\end{abstract}

[Palabras clave: hongos, usos de suelo, siembra directa, agroecosistemas, Pampa Argentina]

Aвstract. Arbuscular mycorrhizal colonization potential varies between agricultural practices and the areas of the Pampas region. The evaluation of the fungal communities requires the identification of the present species, the quantification of the density of propagules and also the determination of their infectivity. In this study, arbuscular mycorrhizal colonization potential (MCP) was evaluated through an experimental trial. Three soils with different land uses (natural grassland, crop rotation and soybean monoculture in direct seeding) from four sites located in different geographical areas of the Pampas Region (Bengolea, Monte Buey, Pergamino and Viale) were used as inoculum using Vicia villosa Roth. as a control plant. The land uses and geographical areas had an effect on the PCM. Monoculture, as rotation scheme, showed greater potential to colonize the roots of Vicia villosa, with respect to natural grassland and crop rotation in most of the proposed combinations, although in previous work we had found that monoculture reduces the richness of the same mycorrhizal communities determined by density and diversity of spores in these soils. These results suggest the limitations of the paradigms of interpretation of the symbiotic systems generated from trials under controlled conditions and a great lack of knowledge of the functioning of plant-microorganism interactions in the soil. Alternatively, the result could be the consequence of an inhibitory effect of the greater fertility N-P in the soils with crop rotation with respect to the soils under monoculture due to a management of historical replenishment of nutrients by fertilization, absent in the cases of monoculture. The use of a single plant species in infectivity assays could also be biasing the results and limiting the expression thereof, by host specificity, in the entire soil mycorrhizal community.

[Keywords: fungi, land uses, no tillage systems, agroecosystems, Argentinean Pampas]

Editor asociado: Gervasio Piñeiro
Recibido: 5 de enero de 2018

Aceptado: 3 de octubre de 2018 


\section{INTRODUCCIÓN}

Los cambios en el uso de la tierra serían la principal amenaza para la biodiversidad (Sala et al. 2000). Recientemente, la aplicación de un meta-análisis permitió demostrar que, a escala local, la conversión y / o la degradación de los ecosistemas promueven una disminución media de $8.1 \%$ de la riqueza de especies, tanto por encima como por debajo de la superficie del suelo (Newbold et al. 2015). Esa disminución de diversidad y, en particular, de la comunidad microbiana del suelo, afecta procesos esenciales como el ciclado de nutrientes y la formación y el mantenimiento de la estructura del suelo, entre otros (Wardle 2006; van der Heijden et al. 2008).

Entre los organismos subterráneos ampliamente distribuidos se encuentran los hongos micorrícico-arbusculares (HMA, Glomeromycota), que se asocian con las raíces del $80 \%$ de las especies de plantas, a las que le proveen acceso a nutrientes y protección contra diversos tipos de estrés (Smith and Read 2008). Los HMA son afectados por diferentes tipos de usos de la tierra, tales como la fragmentación y los cambios en la estructura de hábitats (Mangan et al. 2004; Grilli et al. 2014; Soteras et al. 2015), la compactación del suelo (Nadian et al. 1997), los incendios (Aguilar Fernández et al. 2009; Longo et al. 2014), el pastoreo (Lugo and Cabello 2002) y las prácticas agrícolas (Jansa et al. 2006; Oehl et al. 2010; Stürmer and Siqueira 2011; Cofré et al. 2017). Numerosos estudios documentaron el efecto de las prácticas de manejo agrícolas, tales como el tipo de labranza, la rotación de cultivos, el control de malezas y enfermedades, y el manejo de nutrientes sobre las comunidades de HMA (e.g., Jansa et al. 2006; Alguacil et al. 2008). En términos generales, la labranza convencional, el monocultivo y la fertilización intensa promueven cambios significativos en la composición de las comunidades de HMA al reducir su diversidad (Oehl et al. 2010; Jansa et al. 2014). En términos comparativos, las prácticas agrícolas conservacionistas, como la siembra directa (SD), acompañada de rotación de cultivos y disminución de agroquímicos, tienen efectos más atenuados sobre los propágulos fúngicos (Vestberg et al. 2005), la esporulación de HMA (Karasawa et al. 2002) y la colonización micorrícica (Miller 2000). En el largo plazo, la rotación de cultivos promueve una mayor riqueza de especies de HMA debido a las asociaciones preferenciales entre las diferentes especies de cultivos y hongos (Jansa et al. 2003; Yang et al. 2012). En contraste, los monocultivos tienden a seleccionar HMA específicos que tienden a ser mutualistas inferiores, es decir, menos beneficiosos para sus hospedantes (Johnson et al. 1992). En este contexto, los efectos de las relaciones simbióticas entre plantas y HMA fueron muy estudiadas, desde beneficiosas hasta antagonistas (Johnson et al. 1997; Jones and Smith 2004), según la especie vegetal (Pérez and Urcelay 2009), de qué variedad se trate, de la disponibilidad de nutrientes del suelo, de la identidad planta-hongo (Lehmann et al. 2012), de la combinación de taxones de HMA (Maherali and Klironomos 2007) y de la infectividad micorrícica del suelo (Cuenca et al. 2003).

Si bien existen trabajos acerca de las comunidades de HMA en suelos agrícolas argentinos bajo SD (Menéndez et al. 2001; Schalamuk et al. 2006; Schalamuk and Cabello 2010a,b; Faggioli and Cabello 2013; Imaz et al. 2014; Druille et al. 2015; Cofré et al. 2017), no hay estudios del efecto de distintas prácticas en SD sobre el potencial de colonización micorrícica (PCM). El PCM se refiere a la capacidad que tiene un suelo de iniciar la colonización micorrícica en una planta hospedadora (Plenchette et al. 1989; Martínez and Johnson 2010) a partir de los diferentes propágulos (i.e., esporas, fragmentos de raíces colonizados e hifas de micelio extraradical) que conforman el inóculo de HMA en el suelo (Smith and Read 2008). A su vez, la composición de comunidades de hongos dentro de las raíces suele diferir de la expresada en el banco de esporas (Sanders 2004). Distintas especies de HMA muestran diferentes estrategias (e.g., Maherali and Klironomos 2007; Chagnon et al. 2013) que se ven reflejadas en la producción de esporas en el suelo y en distintos patrones de colonización dentro de la raíz (Hart and Reader 2002; Klironomos and Hart 2002). Estos tipos de inóculos presentan distintas capacidades infectivas para producir nuevas generaciones de esporas (Schalamuck and Cabello 2010). En este sentido, las esporas en suelo muestran una diversidad de HMA mayor que las 
que se encuentran colonizando las raíces (Klironomos and Hart 2002; Schalamuck and Cabello 2010). Por otra parte, las dificultades de generar cultivos monospóricos de HMA no permitió desarrollar una comprensión acabada de la especificidad de esta simbiosis en relación a su planta hospedera, a diferencia de otros tipos de simbiosis de gran impacto en la agricultura y los ecosistemas, como es la simbiosis fijadora de nitrógeno (Smith and Read 2008).

A escala regional, recientemente se observó que la rotación de cultivos mantiene una riqueza de esporas de HMA más alta que el monocultivo, incluso cercana a la de ecosistemas de referencia sin cultivos (Cofré et al. 2017). Por lo tanto, cabría esperar patrones similares en el PCM.

El PCM en agroecosistemas ha sido poco evaluado en el mundo (Duponnois et al. 2001; Jansa et al. 2009; Burrows 2014; Gai et al. 2015), y en la Argentina sólo se ha estudiado en ecosistemas boscosos (Irrazabal et al. 2004; Soteras et al. 2014). Los efectos que ocasionan los diferentes manejos de suelo sobre los HMA son contexto dependientes (GonzálezCortés et al. 2012; Hazard et al. 2013; Jansa et al. 2014; Cofré et al. 2017). En este sentido, las situaciones de uso de la tierra que se repiten regionalmente constituyen escenarios ideales para poner a prueba la respuesta del PCM bajo las diferentes prácticas agrícolas enmarcadas en la SD.

En este estudio se evaluó, mediante una aproximación experimental, el PCM de suelos bajo dos prácticas agrícolas dentro de la SD: monocultivo de soja (MC) y rotación de cultivo (RC), y un ecosistema de referencia, pastizal natural (PN), en cuatro sitios dentro de diferentes áreas geográficas de la Pampa Argentina (Bengolea, Monte Buey, Pergamino y Viale). A su vez, se cuantificaron e identificaron las esporas de HMA provenientes de los mismos suelos (usos y sitios de las distintas áreas geográficas) y se agrupó esos datos a nivel de familia, ya que se considera que las características funcionales de los HMA son filogenéticamente conservadas. Se seleccionó a Vicia villosa Roth. (Fabaceae) como especie trampa en los ensayos de PCM ya que se la emplea como cultivo de cobertura por sus importantes funciones (en los agroecosistemas de las regiones templadas del mundo se la utiliza como pastoreo directo, heno, ensilaje, abonos verdes y suplemento proteico) (Renzi 2009).

\section{MATERIALES Y MÉTODOS}

\section{Manejo de suelos y diseño experimental}

El estudio se realizó en cuatro sitios ubicados en diferentes áreas geográficas siguiendo una transecta de $400 \mathrm{~km}$ orientada de oeste a este, con un gradiente de texturas de suelo (suelos arenosos al oeste y arcillosos al este), en el centro de la Pampa Argentina: Bengolea y Monte Buey (provincia de Córdoba), Pergamino (provincia de Buenos Aires) y Viale (provincia de Entre Ríos). En cada uno de los sitios se seleccionaron tres usos de suelo como tratamientos (ver Cofré et al. 2017 para más detalles). Dos usos de suelo se corresponden con diferentes esquemas de rotación bajo siembra directa (SD) en campos productivos, de acuerdo a la definición de buenas prácticas agrícolas de agricultura certificada establecida según la Asociación Argentina de Productores de Siembra Directa (AAPRESID) y por la Organización de Agricultura y Alimento. Específicamente, uno de ellos consiste en buenas prácticas agrícolas basadas en manejo de rotación de cultivo (RC) (soja [Glycine max (L.) Merr.], maíz [Zea mays L.] y trigo [Triticum aestivum L.]), incluyendo cultivos de cobertura (Vicia villosa Roth.). El otro corresponde a baja rotación cercano al monocultivo de soja (MC). En este contexto, las principales diferencias entre rotación y monocultivo se basan en una mayor proporción de soja en los MC y una proporción más balanceada de maíz, trigo y soja en los campos con RC, incluyendo en esta última cultivos de cobertura invernales, lo que resulta en un mayor rendimiento de los diferentes cultivos. Suelos provenientes de pastizales naturales (PN) cercanos a los campos cultivados se utilizaron como tratamientos de referencia porque no fueron cultivados por lo menos durante los últimos 30 años. Las plantas dominantes presentes en estos pastizales fueron: Andropogon ternatus (Spreng.) Nees, Aristida adscensionis L., Bromus catharticus Vahl., Paspalum dilatatum Poir., P. notatum Flüggé, Poa ligularis Nees ex Steud, Setaria vaginata Spreng. y Stipa speciosa Trin. and Rupr.

En la Tabla 1 se detalla la ubicación, el clima, la textura y las principales características químicas de cada uno de los sitios en cada área geográfica. Para más información de cada uso de suelo y sitios de cada área geográfica, ver Figuerola et al. (2012), Duval et al. (2013) y Cofré et al. (2017). 
En septiembre de 2010, se colectaron tres muestras de suelo (repeticiones) a $10 \mathrm{~cm}$ de profundidad por medio de un barreno de metal (10 cm de diámetro), para cada combinación de uso de suelo/sitio. Cada muestra consistió de 20 submuestras colectadas al azar dentro de un área de $5 \mathrm{~m}^{2}$, que se mezclaron para obtener una muestra compuesta. Las muestras estuvieron separadas por lo menos $50 \mathrm{~m}$ unas de otras. La superficie de los campos bajo PN, RC y MC en cada área comprende entre 2 a 4 ha. Las muestras se colectaron en el momento en que todos los lotes bajo siembra directa se encontraban con rastrojo o cultivos de verano. Por lo tanto, la comunidad de HMA en el suelo presente en las diferentes formas de propágulos (i.e., raíces, hifas y esporas) es la que está disponible para colonizar los cultivos de verano de importancia agrícola (soja y maíz, en estos casos).

En un trabajo previo realizado en estos mismos suelos (Cofré et al. 2017) se identificaron en base a caracteres morfológicos 36 morfoespecies distribuidas en 7 familias: Acaulosporaceae (6), Archaeosporaceae (1), Claroideoglomeraceae (3), Entrophosporaceae (1), Gigasporaceae (11), Glomeraceae (13) y Pacisporaceae (1). Se obtuvieron los datos de densidad total de esporas de cada familia para correlacionarlos con los de PCM.

\section{Determinación del potencial de colonización micorrícica de cada suelo}

El suelo que se usó como inóculo consistió en un homogeneizado de las tres muestras de suelo recolectadas en cada uno de los usos (PN, RC y MC) de cada sitio (Bengolea, Monte Buey, Pergamino y Viale). Los suelos fueron tamizados (malla $1 \mathrm{~cm}$ de diámetro) a fin de remover hojarasca, restos de rastrojo, piedras y palos pequeños. Se realizaron tres diluciones (1:2, 1:8 y 1:32) de cada homogenizado del suelo con perlita y vermiculita esterilizadas ( $1 \mathrm{~h}$ en autoclave a $100{ }^{\circ} \mathrm{C}$ y 1,5 atm de presión) (Díaz and Honrubia 1993). En los resultados se analizan las diluciones 1:2 y 1:32 como situaciones contrastantes. La mezcla de suelo fue transferida a macetas de $400 \mathrm{~cm}^{3}$ y se realizaron 12 repeticiones por dilución por inóculo (324 macetas).

Las semillas de $V$. villosa se esterilizaron superficialmente en $\mathrm{NaClO}$ al $10 \%$, y se las lavó con agua estéril. Una vez crecidas en arena esterilizada, se trasplantaron dos plántulas a cada maceta. El experimento se mantuvo en invernadero $\left(22 / 19{ }^{\circ} \mathrm{C}\right.$ día $/$ noche, fotoperíodo de 14/10 h día/noche) y las plantas se regaron por aspersión durante 1 minuto dos veces al día. Para evaluar el PCM mediante el estado de micorrización, para cada dilución, uso de suelo y área geográfica se cosecharon las plantas de 4 macetas a los 15,30 y 60 días posteriores al inicio del ensayo a fin de obtener una cinética en el tiempo. Los sistemas radicales de las plantas se lavaron y tiñeron siguiendo la técnica de Grace y Stribley (1991) y Brundrett et al. (1996). Las raíces se clarificaron con $\mathrm{KOH} 10 \%, 10$ minutos a 90 ${ }^{\circ} \mathrm{C}$. Posteriormente, se lavaron y acidificaron en HCL por 1 minuto para luego teñirlas con

Tabla 1. Ubicación, clima, textura y clasificación de los suelos de cada uno de los sitios ubicado en cada área geográfica de la Pampa Argentina. Propiedades químicas de cada suelo para los diferentes usos: pastizal natural (PN), rotación de cultivo (RC) y monocultivo de soja (MC) en cada área geográfica.

Table 1. Location, climate, texture and soil classifications at each site located in different geographical areas of the Argentinean Pampas. Chemical properties of soils according to different cropping systems: natural grassland (NG), crop rotation (CR) and soya bean monoculture (MC).

\begin{tabular}{|c|c|c|c|c|c|c|c|c|c|c|c|c|}
\hline & \multicolumn{3}{|c|}{ Bengolea } & \multicolumn{3}{|c|}{ Monte Buey } & \multicolumn{3}{|c|}{ Pergamino } & \multicolumn{3}{|c|}{ Viale } \\
\hline & PN & $\mathrm{RC}$ & MC & $\mathrm{PN}$ & $\mathrm{RC}$ & $\mathrm{MC}$ & PN & $\mathrm{RC}$ & $\mathrm{MC}$ & PN & $\mathrm{RC}$ & MC \\
\hline Latitud (S) & \multicolumn{3}{|c|}{$33^{\circ} 01^{\prime} 31^{\prime \prime}$} & \multicolumn{3}{|c|}{$32^{\circ} 58^{\prime} 14^{\prime \prime}$} & \multicolumn{3}{|c|}{$33^{\circ} 56^{\prime} 36^{\prime \prime}$} & \multicolumn{3}{|c|}{$31^{\circ} 52^{\prime} 59^{\prime \prime}$} \\
\hline Longitud $(\mathrm{O})$ & \multicolumn{3}{|c|}{$63^{\circ} 37^{\prime} 53^{\prime \prime}$} & \multicolumn{3}{|c|}{$62^{\circ} 27^{\prime} 06^{\prime \prime}$} & \multicolumn{3}{|c|}{$60^{\circ} 33^{\prime} 57^{\prime \prime}$} & \multicolumn{3}{|c|}{$59^{\circ} 40^{\prime} 07^{\prime \prime}$} \\
\hline Elevación (m s. n. m) & \multicolumn{3}{|c|}{231} & \multicolumn{3}{|c|}{110} & \multicolumn{3}{|c|}{56} & \multicolumn{3}{|c|}{91} \\
\hline Precipitación anual (mm) & \multicolumn{3}{|c|}{880} & \multicolumn{3}{|c|}{930} & \multicolumn{3}{|c|}{1000} & \multicolumn{3}{|c|}{1156} \\
\hline Temperatura media anual $\left({ }^{\circ} \mathrm{C}\right)$ & \multicolumn{3}{|c|}{17} & \multicolumn{3}{|c|}{17} & \multicolumn{3}{|c|}{16} & \multicolumn{3}{|c|}{18} \\
\hline Textura & \multicolumn{3}{|c|}{ Franco arenoso } & \multicolumn{3}{|c|}{ Franco limoso } & \multicolumn{3}{|c|}{ Franco limoso } & \multicolumn{3}{|c|}{$\begin{array}{c}\text { Arcillo limoso / } \\
\text { Franco arcillo limoso }\end{array}$} \\
\hline Tipo de suelo & \multicolumn{3}{|c|}{ Haplustol éntico } & \multicolumn{3}{|c|}{ Argiudol típico } & \multicolumn{3}{|c|}{ Argiudol típico } & \multicolumn{3}{|c|}{ Argiudol vértico } \\
\hline Carbono orgánico total (COT, \%) & $\begin{array}{c}1.81 \pm \\
0.1\end{array}$ & $\begin{array}{l}1.57 \pm \\
0.04\end{array}$ & $\begin{array}{c}1.28 \pm \\
0.1\end{array}$ & $\begin{array}{c}3.58 \pm \\
0.36\end{array}$ & $\begin{array}{l}2.2 \pm \\
0.02\end{array}$ & $\begin{array}{l}1.84 \pm \\
0.03\end{array}$ & $\begin{array}{l}2.86 \pm \\
0.09\end{array}$ & $\begin{array}{c}1.85 \pm \\
0.14\end{array}$ & $\begin{array}{c}1.89 \pm \\
0.04\end{array}$ & $\begin{array}{l}3.31 \pm \\
0.63\end{array}$ & $\begin{array}{l}3.87 \pm \\
0.56\end{array}$ & $\begin{array}{l}2.61 \pm \\
0.07\end{array}$ \\
\hline Nitrógeno total (NT \%) & $\begin{array}{c}0.14 \pm \\
0.01\end{array}$ & $\begin{array}{l}0.12 \pm \\
0.00\end{array}$ & $\begin{array}{l}0.11 \pm \\
0.003\end{array}$ & $\begin{array}{l}0.28 \pm \\
0.02\end{array}$ & $\begin{array}{l}0.17 \pm \\
0.01\end{array}$ & $\begin{array}{c}0.13 \pm \\
0.01\end{array}$ & $\begin{array}{c}0.24 \pm \\
0.01\end{array}$ & $\begin{array}{c}0.16 \pm \\
0.01\end{array}$ & $\begin{array}{l}0.14 \pm \\
0.001\end{array}$ & $\begin{array}{c}0.26 \pm \\
0.05\end{array}$ & $\begin{array}{c}0.29 \pm \\
0.04\end{array}$ & $\begin{array}{c}0.2 \pm \\
0.005\end{array}$ \\
\hline Fósforo disponible (mg/kg, P) & $\begin{array}{l}13.8 \pm \\
2.89\end{array}$ & $\begin{array}{c}7.07 \pm \\
0.73\end{array}$ & $\begin{array}{l}9.63 \pm \\
1.85\end{array}$ & $\begin{array}{c}345.6 \pm \\
61.88\end{array}$ & $\begin{array}{l}71.33 \pm \\
2.45\end{array}$ & $\begin{array}{c}6.27 \pm \\
0.26\end{array}$ & $\begin{array}{l}4.3 \pm \\
1.52\end{array}$ & $\begin{array}{c}11.17 \pm \\
4.83\end{array}$ & $\begin{array}{c}5.13 \pm \\
0.12\end{array}$ & $\begin{array}{l}5.73 \pm \\
1.52\end{array}$ & $\begin{array}{c}27.37 \pm \\
4.39\end{array}$ & $\begin{array}{c}33.33 \pm \\
5.00\end{array}$ \\
\hline $\mathrm{pH}$ & $\begin{array}{l}6.3 \pm \\
0.03 \\
\end{array}$ & $\begin{array}{l}5.8 \pm \\
0.06 \\
\end{array}$ & $\begin{array}{l}5.7 \pm \\
0.00 \\
\end{array}$ & $\begin{array}{l}5.5 \pm \\
0.18 \\
\end{array}$ & $\begin{array}{l}5.6 \pm \\
0.06 \\
\end{array}$ & $\begin{array}{l}6.1 \pm \\
0.00 \\
\end{array}$ & $\begin{array}{c}5.73 \pm \\
0.07 \\
\end{array}$ & $\begin{array}{c}6.1 \pm \\
0.0 \\
\end{array}$ & $\begin{array}{l}6.1 \pm \\
0.00 \\
\end{array}$ & $\begin{array}{c}6.43 \pm \\
0.58 \\
\end{array}$ & $\begin{array}{c}6.83 \pm \\
0.23 \\
\end{array}$ & $\begin{array}{c}5.67 \pm \\
0.13 \\
\end{array}$ \\
\hline
\end{tabular}


azul de anilina al $0.05 \%$ durante 5 minutos a $90{ }^{\circ} \mathrm{C}$. Las raíces teñidas se examinaron bajo lupa estereoscópica Nikon SMZ745T a fin de evaluar la colonización micorrícica arbuscular (CMA) con el método de intersección en gradilla (Giovannetti and Mosse 1980).

\section{Análisis estadístico}

El análisis estadístico de los datos se realizó con el software estadístico InfoStat Plus version 2011 (www.infostat.com.ar). Para todos los datos se comprobaron los supuestos de normalidad y homogeneidad mediante las pruebas de Shapiro-Wilks y Levene, respectivamente. Cuando los datos mostraron una distribución no normal, se trasformaron a $\log 10$. Con los datos obtenidos se realizó un análisis de la varianza (ANAVA) para poner a prueba diferencias del PCM entre los usos de suelo y entre sitios de estudio de las diferentes áreas geográficas. El uso de suelo (con tres niveles: PN, RC y MC) y los sitios en cada área geográfica (con cuatro niveles: Bengolea, Monte Buey, Pergamino y Viale) fueron los factores fijos del modelo. Para examinar las diferencias del PCM entre los usos de suelo dentro de cada sitio, se utilizó el uso de suelo como factor fijo del modelo (con sus tres niveles) para cada una de los sitios.

Se utilizó la prueba a posteriori de Fisher (LSD) $(P<0.05)$ para comparar las medias entre las variables de efecto fijo y sus interacciones. Se calcularon los coeficientes de correlación de Spearman entre las familias de HMA identificadas, las variables químicas de los suelos de la Tabla 1 ( $\mathrm{pH}$, Carbono orgánico total [COT], Nitrógeno total [NT] y Fósforo disponible [P]) y los porcentajes de colonización en las raíces de $V$. villosa.

\section{Resultados}

\section{Potencial de colonización micorrícica, usos de suelo y sitios}

Las raíces de $V$. villosa mostraron las estructuras típicas de los HMA: puntos de entrada, hifas, vesículas y arbúsculos. El PCM se evaluó a través del porcentaje de CMA que osciló entre 0 y $68 \%$ para la dilución 1:2 y entre 0 y $59 \%$ para la dilución 1:32. Para estas dos diluciones de suelo, la CMA presentó diferencias significativas entre los usos a los 30 y 60 días, y entre los cuatro sitios para todas las situaciones planteadas (15, 30 y 60 días) de crecimiento de las plantas de Vicia, con excepción de 1:32 a los 60 días.

Respecto al suelo menos diluido (1:2), la CMA resultó significativa para la interacción usos de suelo y sitios de estudio a los 30 días (Tabla 2). Los valores más altos para esa variable se registraron en PN de Pergamino y los más bajos en MC de Viale. A los 60 días de crecimiento de las plantas los valores más altos se observaron en el MC con respecto a los otros usos de suelo. En cuanto a los sitios, Bengolea y Pergamino registraron los valores más altos, Viale intermedios y Monte Buey los más bajos.

Conla dilución 1:32 la CMA mostrólos valores más altos en los campos bajo monocultivo y los más bajos en rotación (Tabla 2). Entre los sitios de estudio, los valores más altos para esa variable fueron observados en Pergamino y los más bajos en Monte Buey. Se observó interacción significativa entre usos de suelo y sitios a los 30 días. Los valores más altos de CMA se observaron en el MC de Pergamino y los más bajos en Monte Buey.

\section{Potencial de colonización micorrícica y usos de suelo dentro de cada sitio}

La CMA aumentó a lo largo del tiempo para los usos de suelo y sitios de estudio (Figura 1 y material suplementario), con algunas excepciones. Para la dilución 1:2 la CMA presentó diferencias significativas entre los usos de suelo en Bengolea a los 15 días, y en Monte Buey y Viale a los 30 días de crecimiento de las plantas de Vicia. Los valores más altos se observaron para el PN en Bengolea y Viale, y para el MC en Monte Buey.

En el suelo diluido 32 veces, a los 15 días, la CMA mostró diferencias significativas en Viale, con los valores más altos registrados en $\mathrm{RC}$ y los más bajos en MC. A los 30 días de crecimiento, la CMA mostró diferencias significativas entre los usos de suelo en Bengolea, Monte Buey y Pergamino. Para Bengolea, los valores más altos se registraron en PN y los más bajos para RC. En Monte Buey y Pergamino los valores más altos se observaron en MC y los más bajos en RC. Al final del experimento (60 días), la CMA mostró diferencias significativas entre los usos de suelo en Monte Buey, y los valores más altos se observaron en los campos bajo MC con respecto a los otros dos usos de suelo (Figura 1 y material suplementario). 
Tabla 2. Colonización micorrícico-arbuscular (\%) de Vicia villosa para dos diluciones de suelo $(1: 2,1: 32)$ de cada uno de los usos (US) utilizados como inóculo: pastizal natural (PN), rotación de cultivo (RC) y monocultivo (MC), durante tres fechas de cosecha (15, 30 y 60 días a partir del inicio del experimento) en los cuatro sitios (S) de cada área geográfica: Bengolea (B), Monte Buey (MB), Pergamino (P) y Viale (V). Los valores son la media \pm error estándar. Resultados del ANAVA entre usos de suelo, sitios y su interacción. Letras diferentes indican diferencias significativas según la prueba a posteriori LSD Fisher $(P<0.05)$. ns, no significativo; ${ }^{*} P<0.05$; ${ }^{* *} P<0.01 ;{ }^{* * *} P<0.001$.

Table 2. Arbuscular mycorrhizal colonization of Vicia villosa for two soil dilutions (1: 2, 1:32) of each land uses (US) used as inoculum: natural grassland (NG), crop rotation (CR) and monoculture (MC), during three harvest dates $(15,30$ and 60 days from the beginning of the experiment) in the four sites (S) of each geographical area: Bengolea (B), Monte Buey (MB), Pergamino $(\mathrm{P})$ and Viale $(\mathrm{V})$. The values are the mean \pm standard error. Results of ANAVA between land uses, geographical areas and their interaction. Different letters indicate significant differences according to the Fisher LSD a posteriori test $(P<0.05)$. ns, non-significant; ${ }^{*} P<0.05 ; * * P<0.01 ; * * * P<0.001$.

\begin{tabular}{|c|c|c|c|c|c|c|c|c|c|c|}
\hline $\begin{array}{c}\text { Factor } \\
\text { Dilución 1:2 }\end{array}$ & Término & $\begin{array}{l}15 \text { días } \\
\text { me } \pm e e\end{array}$ & $\mathrm{~F}$ & $P$ & $\begin{array}{l}30 \text { días } \\
\text { me } \pm e e\end{array}$ & F & $P$ & $\begin{array}{l}60 \text { días } \\
\text { me } \pm e e\end{array}$ & F & $P$ \\
\hline \multirow[t]{3}{*}{ US } & PN & $11.76 \pm 3.25$ & 0.17 & ns & $24.80 \pm 5.87 \mathrm{a}$ & 3.05 & * & $21.39 \pm 4.91 \mathrm{ab}$ & 3.32 & $*$ \\
\hline & $\mathrm{RC}$ & $10.13+3.23$ & & & $14.75 \pm 2.86 \mathrm{~b}$ & & & $14.12 \pm 2.81 \mathrm{~b}$ & & \\
\hline & $\mathrm{MC}$ & $11.1 \pm 4.37$ & & & $21.29 \pm 3.80 \mathrm{ab}$ & & & $29.05 \pm 4.67$ a & & \\
\hline \multirow[t]{4}{*}{$S$} & B & $2.90 \pm 0.98 \mathrm{c}$ & 36.39 & $* * *$ & $24.67 \pm 4.30 \mathrm{~b}$ & 14.9 & $* * *$ & $29.19 \pm 5.60 \mathrm{a}$ & 3.27 & * \\
\hline & MB & $1.18 \pm 0.98 \mathrm{bc}$ & & & $11.01 \pm 4.09 \mathrm{c}$ & & & $11.48 \pm 4.65 \mathrm{~b}$ & & \\
\hline & $\mathrm{P}$ & $31.23 \pm 3.74 \mathrm{a}$ & & & $36.73 \pm 4.91 \mathrm{a}$ & & & $28.07 \pm 4.45 \mathrm{a}$ & & \\
\hline & $\mathrm{V}$ & $8.66 \pm 2.17 \mathrm{~b}$ & & & $8.72 \pm 2.11 \mathrm{c}$ & & & $17.35 \pm 4.29 \mathrm{ab}$ & & \\
\hline \multirow[t]{12}{*}{$\mathrm{US}^{*} \mathrm{~S}$} & $\mathrm{PN}^{*} \mathrm{~B}$ & $5.98 \pm 2.18 \mathrm{bc}$ & 1.11 & ns & $36.61 \pm 10.59 \mathrm{ab}$ & 3.23 & $* *$ & $29.22 \pm 13.84$ & 0.36 & ns \\
\hline & $\mathrm{PN}^{*} \mathrm{MB}$ & $0.16 \pm 0.16 \mathrm{c}$ & & & $2.50 \pm 0.53 \mathrm{e}$ & & & $4.18 \pm 0.99$ & & \\
\hline & $\mathrm{PN}^{*} \mathrm{P}$ & $28.71 \pm 6.74 \mathrm{a}$ & & & $44.27 \pm 13.74 \mathrm{a}$ & & & $32.28 \pm 8.08$ & & \\
\hline & $\mathrm{PN}^{*} \mathrm{~V}$ & $12.17 \pm 3.12 \mathrm{~b}$ & & & $15.82 \pm 4.61 \mathrm{cde}$ & & & $19.86 \pm 8.01$ & & \\
\hline & $\mathrm{RC}^{*} \mathrm{~B}$ & $1.89 \pm 0.72 \mathrm{bc}$ & & & $21.70 \pm 3.50 \mathrm{bcd}$ & & & $24.29 \pm 7.01$ & & \\
\hline & $\mathrm{RC}^{*} \mathrm{MB}$ & $0.40 \pm 0.40 \mathrm{c}$ & & & $2.60 \pm 1.06 \mathrm{e}$ & & & $5.32 \pm 1.10$ & & \\
\hline & $\mathrm{RC}^{*} \mathrm{P}$ & $27.97 \pm 4.59$ a & & & $28.17 \pm 1.26 \mathrm{abc}$ & & & $17.18 \pm 4.99$ & & \\
\hline & $\mathrm{RC}^{*} \mathrm{~V}$ & $10.24 \pm 5.20 \mathrm{bc}$ & & & $6.52 \pm 0.75$ de & & & $9.69 \pm 3.54$ & & \\
\hline & $M C^{*} B$ & $0.83 \pm 0.53 \mathrm{bc}$ & & & $15.70 \pm 1.09 \mathrm{cde}$ & & & $34.5 \pm 9.39$ & & \\
\hline & $\mathrm{MC}^{*} \mathrm{MB}$ & $2.98 \pm 2.98$ bc & & & $27.92 \pm 6.27 \mathrm{abc}$ & & & $24.92 \pm 12.06$ & & \\
\hline & $M C^{*} \mathrm{P}$ & $37.00 \pm 8.42 \mathrm{a}$ & & & $37.74 \pm 5.49 \mathrm{ab}$ & & & $34.75 \pm 8.22$ & & \\
\hline & $\mathrm{MC}^{*} \mathrm{~V}$ & $3.57 \pm 1.22$ bc & & & $3.82 \pm 0.94 \mathrm{e}$ & & & $22.49 \pm 9.77$ & & \\
\hline \multicolumn{11}{|c|}{ Dilución 1:32 } \\
\hline \multirow[t]{3}{*}{ US } & PN & $4.75 \pm 1.92$ & 0.12 & ns & $8.23 \pm 1.65 b$ & 7.11 & $* *$ & $12.75 \pm 3.57 \mathrm{~b}$ & 3.86 & $*$ \\
\hline & $\mathrm{RC}$ & $4.44 \pm 1.54$ & & & $6.41 \pm 5.50 \mathrm{~b}$ & & & $9.33 \pm 9.88 \mathrm{~b}$ & & \\
\hline & $\mathrm{MC}$ & $3.96 \pm 2.13$ & & & $13.73 \pm 3.92 \mathrm{a}$ & & & $20.22 \pm 9.39$ a & & \\
\hline \multirow[t]{4}{*}{ S } & B & $0.26 \pm 0.26 b$ & 25.27 & $* * *$ & $9.51 \pm 1.29 \mathrm{~b}$ & 20.06 & $* * *$ & $16.31 \pm 3.42$ & 1.29 & ns \\
\hline & MB & $0.00 \pm 0.00 \mathrm{~b}$ & & & $3.14 \pm 1.02 \mathrm{c}$ & & & $8.62 \pm 2.47$ & & \\
\hline & $\mathrm{P}$ & $14.37 \pm 2.39 \mathrm{a}$ & & & $19.82 \pm 4.46 \mathrm{a}$ & & & $16.53 \pm 3.12$ & & \\
\hline & V & $2.91 \pm 1.01 \mathrm{~b}$ & & & $5.37 \pm 1.53 \mathrm{c}$ & & & $14.94 \pm 4.53$ & & \\
\hline \multirow[t]{12}{*}{ US*S } & $\mathrm{PN}^{*} \mathrm{~B}$ & $0.78 \pm 0.78 \mathrm{c}$ & 0.8 & ns & $13.99 \pm 1.10 \mathrm{~b}$ & 8.41 & $* * *$ & $14.17 \pm 5.67$ & 0.94 & ns \\
\hline & $\mathrm{PN}^{*} \mathrm{MB}$ & $0.00 \pm 0.00 \mathrm{c}$ & & & $0.00 \pm 0.00 \mathrm{e}$ & & & $2.13 \pm 0.22$ & & \\
\hline & $\mathrm{PN}^{*} \mathrm{P}$ & $15.41 \pm 4.60 \mathrm{a}$ & & & $11.42 \pm 3.36 \mathrm{bc}$ & & & $12.38 \pm 2.50$ & & \\
\hline & $\mathrm{PN}^{*} \mathrm{~V}$ & $2.82 \pm 0.61 \mathrm{c}$ & & & $7.51 \pm 2.23$ bcdef & & & $22.33 \pm 12.16$ & & \\
\hline & $\mathrm{RC}^{*} \mathrm{~B}$ & $0.00 \pm 0.00 \mathrm{c}$ & & & $4.89 \pm 1.19$ cdef & & & $13.46 \pm 7.23$ & & \\
\hline & $\mathrm{RC}^{*} \mathrm{MB}$ & $0.00 \pm 0.00 \mathrm{c}$ & & & $2.90 \pm 1.99 \mathrm{def}$ & & & $4.49 \pm 1.56$ & & \\
\hline & $\mathrm{RC}^{*} \mathrm{P}$ & $11.87 \pm 3.18 \mathrm{ab}$ & & & $10.98 \pm 2.68 \mathrm{bcd}$ & & & $14.07 \pm 5.88$ & & \\
\hline & $\mathrm{RC}^{*} \mathrm{~V}$ & $5.91 \pm 2.24 \mathrm{bc}$ & & & $6.88 \pm 3.64$ bcdef & & & $5.29 \pm 2.53$ & & \\
\hline & $M C^{*} B$ & $0.00 \pm 0.00 \mathrm{c}$ & & & $9.65 \pm 1.39$ bcde & & & $21.32 \pm 5.63$ & & \\
\hline & $\mathrm{MC}^{*} \mathrm{MB}$ & $0.00 \pm 0.00 \mathrm{c}$ & & & $6.52 \pm 0.59$ bcdef & & & $19.23 \pm 2.76$ & & \\
\hline & $M C^{*} \mathrm{P}$ & $15.85 \pm 5.32 \mathrm{a}$ & & & $37.04 \pm 7.20 \mathrm{a}$ & & & $23.14 \pm 6.61$ & & \\
\hline & $\mathrm{MC}^{*} \mathrm{~V}$ & $0.00 \pm 0.00 \mathrm{c}$ & & & $1.71 \pm 0.83$ ef & & & $17.20 \pm 4.54 \mathrm{abc}$ & & \\
\hline
\end{tabular}

\section{Análisis de correlación}

La CMA mostró correlación significativa y positiva para ambas diluciones $(1: 2,1: 32)$ a los 15 días, con las familias Entrophosporaceae,
Glomeraceae y con Acaulosporaceae sólo con el suelo más diluido (1:32) (Tabla 3). Para ambas diluciones de suelo, a los 30 días, la CMA fue positiva con Acaulosporaceae, y con Entrophosporaceae solo con el suelo 


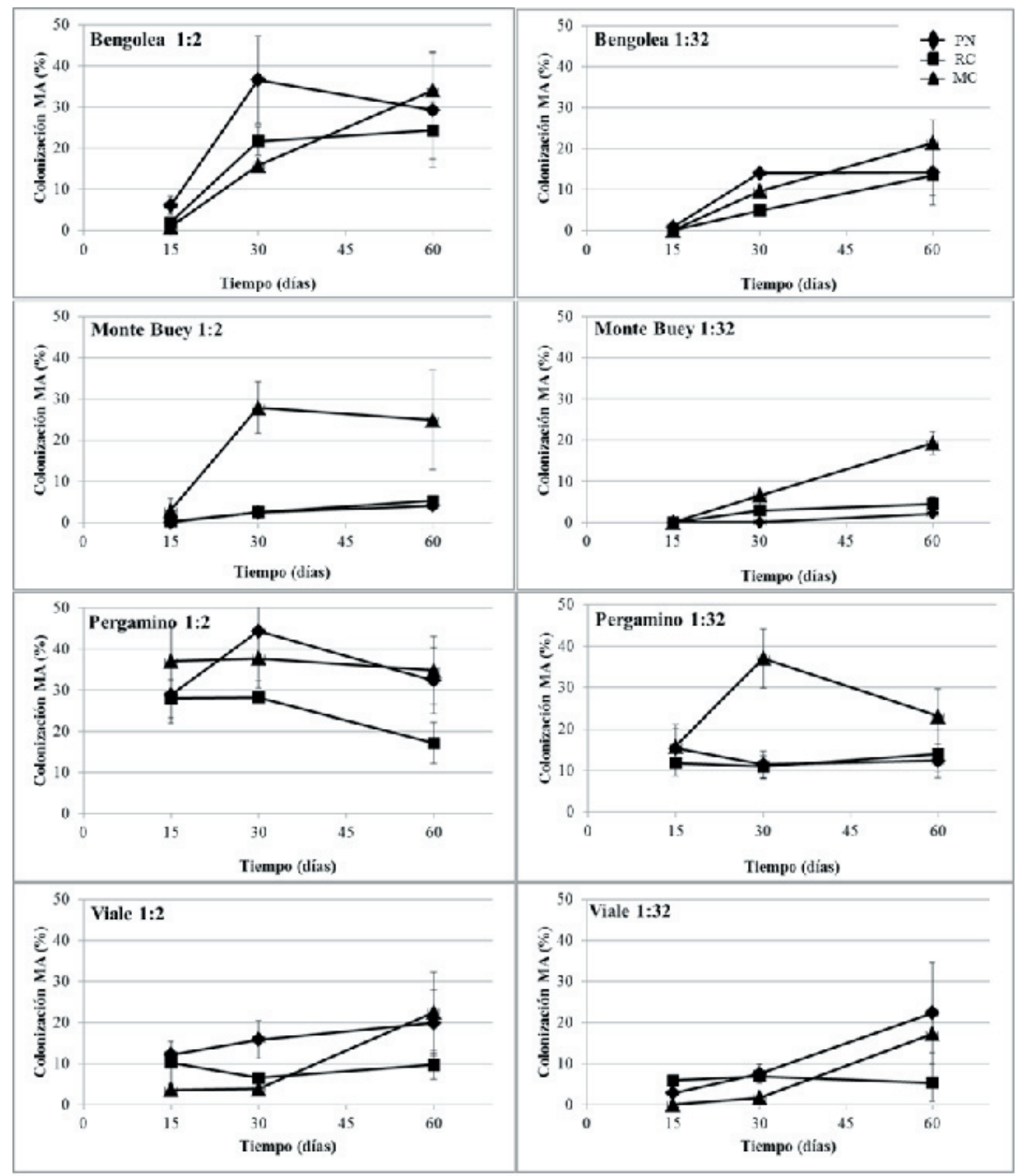

Figura 1. Colonización micorrícico-arbuscular (\%) de las plantas de Vicia villosa crecidas en tres suelos con diferentes usos (pastizal natural $[\mathrm{PN}]$, rotación de cultivo $[\mathrm{RC}]$ y monocultivo $[\mathrm{MC}]$ ) en dos diluciones (1:2 y 1:32) del suelo durante tres fechas de cosecha (15, 30 y 60 días a partir del inicio del experimento) para los cuatro sitios en cada área geográfica. Los valores son la media \pm error estándar $(n=4)$.

Figure 1. Arbuscular mycorrhizal fungi (AMF) colonization of Vicia villosa seedlings grown in three soils with different uses, natural grassland (NG), crop rotation (CR) and monoculture (MC). in two soil dilutions (1:2 and 1:32) during three harvesting dates $(15,30$ and 60 days from the beginning of the experiment) for four sites in each geographical area. The values are the mean \pm standard error $(n=4)$.

más diluido. A los 60 días, la dilución 1: 2 presentó relaciones positivas con las familias Acaulosporaceae y Pacisporaceae. Correlaciones fuertemente negativas se presentaron entre la CMA y el fósforo disponible (P), el Carbono orgánico total (COT) y el Nitrógeno total (NT). Entre la CMA y el P esos datos se presentaron en ambas diluciones de suelo y en todos los tiempos de cosecha, entre la CMA y COT y el NT a los 30 y 60 días en la dilución 1:2 y a los 60 días en la dilución 1:32. La CMA se correlacionó positivamente con el pH a los 30 días para la dilución 1:32 (Tabla 3). 
Tabla 3. Análisis de correlación de Spearman entre los porcentajes de colonización de las raíces de Vicia villosa (crecidas en las diferentes diluciones de suelo (1:2 y 1:32) y durante las tres fechas de cosecha (15, 30 y 60 días a partir del inicio del experimento)) y las distintas familias de hongos micorrícico-arbusculares (HMA) identificadas y las variables químicas de los suelos (Carbono orgánico total, COT; Nitrógeno total, NT; Fósforo disponible, $P$ ). ${ }^{*} P<0.05 ;{ }^{* *} P<0.01$; ${ }^{* * * P<0.001 .}$

Table 3. Spearman correlation analysis between the colonization percentages of Vicia villosa roots grown in the different soil dilutions (1:2 y 1:32) and during three harvesting dates (15, 30 and 60 days from the beginning of the experiment) and the different families of arbuscular mycorrhizal fungi (AMF) identified and chemical variables of soils (total organic Carbon,COT; total Nitrogen, NT; available Phosphorus, P). ${ }^{*} P<0.05 ;{ }^{* *} P<0.01$; ${ }^{* * *} P<0.001$.

\begin{tabular}{lllllll}
\hline Colonización según dilución (días) & $1: 2(15)$ & $1: 2(30)$ & $1: 2(60)$ & $1: 32(15)$ & $1: 32(30)$ & $1: 32(60)$ \\
\hline Familia de HMA/variables químicas & & & & & & \\
Acaulosporaceae & 0.51 & $0.71^{*}$ & $0.63^{*}$ & $0.55^{*}$ & $0.81^{* *}$ & 0.38 \\
Archaeosporaceae & 0.08 & -0.38 & -0.27 & $-3.00 \mathrm{E}-03-0.3$ & -0.16 \\
Claroideoglomeraceae & 0.39 & 0.27 & 0.24 & 0.38 & 0.45 & 0.22 \\
Entrophosporaceae & $0.58^{*}$ & 0.52 & 0.43 & $0.64^{*}$ & $0.69^{* *}$ & 0.16 \\
Glomeraceae & $0.65^{*}$ & 0.44 & 0.15 & $0.58^{*}$ & 0.5 & 0.17 \\
Gigasporaceae & -0.06 & 0.36 & 0.49 & 0.03 & 0.32 & -0.05 \\
Pacisporaceae & 0.41 & 0.41 & $0.6^{*}$ & 0.42 & 0.53 & 0.45 \\
COT & 0,15 & $-0.34^{*}$ & $-0.46^{* *}$ & 0.23 & -0.26 & $-0.41^{*}$ \\
pH & 0.26 & 0.28 & 0.31 & 0.26 & $0.33^{*}$ & 0.22 \\
NT & 0.22 & $-0.34^{*}$ & $-0.48^{* *}$ & 0.30 & -0.26 & $-0.43^{* *}$ \\
P & $-0.50^{* *}$ & $-0.76^{* * *}$ & $-0.69^{* * *}$ & $-0.50^{* *}$ & $-0.59^{* * *}$ & $-0.46^{* *}$ \\
\hline
\end{tabular}

\section{Discusión}

En este trabajo se evaluó el potencial de colonización micorrícica (PCM) en suelos provenientes de diferentes sistemas de cultivo: monocultivo de soja $(\mathrm{MC})$, rotación de cultivo (RC) y un pastizal natural (PN) como referencia, en 4 sitios ubicados en diferentes áreas geográficas de la Pampa Argentina: Bengolea y Monte Buey (provincia de Córdoba), Pergamino (provincia de Buenos Aires) y Viale (provincia de Entre Ríos). Los resultados muestran actividad micorrícica arbuscular en la mayoría de los casos analizados. El PCM presentó valores de 0 a $68 \%$, similares a los observados en estudios previos (Purin et al. 2006; Bedini et al. 2013; Soteras et al. 2014; Gai et al. 2015). Además, el PCM resultó diferente entre los manejos de suelo para ambas diluciones (1:2 y 1:32) y entre los diferentes sitios localizados en las distintas áreas geográficas. Sin embargo, los patrones de variación no resultaron consistentes con lo observado en las comunidades de esporas (Cofré et al. 2017). Para la mayoría de las combinaciones, el PCM de las raíces de $V$. villosa se incrementó con el tiempo excepto Viale, mostrando los valores más altos en MC a los 60 días de crecimiento de las plantas. En esta línea, se observaron altos valores de PCM en bosques degradados del centro de la Argentina (Soteras et al. 2014). Por otro lado, Purin et al. (2006) observaron mayores PCM en pastizales utilizados como control que en plantaciones de manzana bajo agricultura orgánica o labranza convencional al sur de Brasil. En conjunto, estos resultados muestran que el efecto de los usos de la tierra sobre el PCM varía con el sistema estudiado y el tipo de uso. El PCM varió entre los sitios de estudio, lo que es consistente con lo observado en la composición de las comunidades HMA (Cofré et al., 2017). Es posible que otros factores como la historia de uso de los suelos, las propiedades físico-químicas propias de cada sitio y el "provincialismo" de los microorganismos del suelo estén influyendo estos patrones de respuesta (Hedlund et al. 2003; Ge et al. 2008).

Los agroecosistemas poseen características particulares que influyen en la actividad de los HMA. Mientras que en ecosistemas naturales existen diferentes especies de plantas de distintas edades y estacionalidad hospedando los HMA, en sistemas agrícolas como es el caso de los campos bajo RC y MC en este estudio, se observan dos períodos estacionales muy marcados: una parte del año (febrero) en la que se registra una gran densidad de plantas hospedantes pertenecientes a la misma especie vegetal, y otra parte del año (septiembre) con ausencia de hospedantes o, en algunos casos, con presencia de cultivos de cobertura o cultivos de invierno. Los resultados de este trabajo se enmarcan dentro de un muestreo, en una estación del año (primavera) y en una especie de planta, y muestran la infectividad que tienen los suelos previos a la siembra de la soja. 
En estos mismos sitios de muestreo, Cofré et al. (2017) observaron que la densidad de esporas fue mayor en el pastizal natural que en los campos bajo siembra directa (MC y RC), excepto para Monte Buey. La riqueza mostró un patrón similar sólo en Bengolea y no se observaron diferencias en los otros sitios de estudio. En el presente trabajo, los patrones de infectividad mostraron en general valores más altos en MC lo que sugiere que no existe una relación directa entre la densidad de esporas de HMA y la infectividad del suelo medido con una única especie hospedera ( $V$. villosa). Es posible que la infectividad se relacione con alguna especie o conjunto de ellas más que con la densidad total o la riqueza. En este sentido, los análisis de correlación muestran una relación positiva del PCM con las familias Entrophosporaceae y Glomeraceae en las dos diluciones y con Acaulosporaceae en una de ellas. De estas tres familias, Glomeraceae es la que promueve mayores porcentajes de colonización micorrícica y menos beneficios en términos de crecimiento y nutrición (Maherali and Klironomos 2007, 2012). Por lo tanto, las implicancias funcionales de los PCM observadas en este trabajo deberían ser evaluadas experimentalmente para cada una de las situaciones de uso. De este modo se podría establecer la relevancia de las diferencias en la composición de esporas y del PCM en el crecimiento y nutrición de la soja. Sería interesante detectar, mediante técnicas moleculares, los HMA que se encuentran colonizando las raíces de las plantas de estudio (Redecker et al. 2000; Davison et al. 2012), ya que las especies de la rizosfera no necesariamente reflejan los simbiontes radicales (Clapp et al. 1995). En estudios previos se observó que la colonización micorrícica no necesariamente se relaciona con el beneficio proporcionado por los HMA hacia la planta (Gange and Ayres 1999; Smith and Read 2008), por lo que una especie de planta puede tener altos porcentajes de colonización micorrícica arbuscular sin que los HMA con los que se asocia les brinden algún beneficio (Johnson et al. 1997).

Es importante notar las diferencias en la fertilización histórica de $\mathrm{P}$ y $\mathrm{N}$ en los campos bajo RC y MC (ver Duval et al. 2015), donde los mayores valores se observan en los campos sujetos a rotación. Esto podría explicar en parte los bajos valores de colonización observados en $V$. villosa para esos mismos campos, ya que en general existe una relación negativa entre la fertilidad del suelo y la colonización micorrícica (Treseder 2004). Los análisis de correlación negativos entre la CMA y el $\mathrm{P}$, el COT y el $\mathrm{N}$ son consistentes con esta afirmación. Más aun, los suelos del PN y los campos bajo RC en Monte Buey mostraron los valores más altos de $\mathrm{P}$ disponible (Tabla 1) que las otras combinaciones de usos de suelo y áreas geográficas. Estos altos valores de $\mathrm{P}$ disponible se pueden explicar porque dichos lotes poseen una historia previa de cría de ganado porcino, actividad que genera grandes cantidades de $\mathrm{P}$ orgánico que con el tiempo puede mineralizarse y dar lugar a concentraciones elevadas de $\mathrm{P}$ disponible (Jorge Romagnoli, Establecimiento La Lucia, Monte Buey, comunicación personal; Luis Wall, datos no publicados). En este trabajo y en Cofré et al. (2017) se observaron los valores más bajos de CMA, densidad y riqueza de esporas de HMA en el PN seguido por RC en Monte Buey.

Finalmente, los resultados aquí presentados, junto con las observaciones realizadas previamente sobre la densidad y la diversidad de esporas de HMA en estos mismos suelos (Cofré et al. 2017) señalan las limitaciones del paradigma actual de las simbiosis de HMA. El conocimiento de estas simbiosis se desarrolló históricamente sobre la base de ensayos en condiciones controladas y que se interpelan frente a la complejidad de la estructura y dinámica de los microbiomas actualmente reconocidos en los suelos (Figuerola et al. 2014). Tal como lo señalan Ryan and Graham (2018), los resultados obtenidos en experimentos de invernadero generalmente no son aplicables al campo, ya que no imitan las condiciones que ocurren en la naturaleza (e.g., ausencia de condiciones extremas o variaciones ambientales, el efecto de la esterilización del suelo sobre los microorganismos y los nutrientes, uso de una única o pocas plantas, limitación del espacio para que se desarrollen las raíces, entre otras).

\section{Conclusión}

Este trabajo sugiere que los campos bajo monocultivo de soja del centro de la Argentina presentan un mayor potencial de colonización micorrícica en plantas de Vicia villosa, aunque las diferencias significativas mostraron excepciones. Los patrones de variación de la colonización observados en $V$. villosa fueron diferentes a los de las otras variables analizadas (densidad y riqueza de 
esporas) para la comunidad de HMA en los mismos sitios (Cofré et al. 2017). Posiblemente, una o varias especies de HMA estén jugando roles específicos en promover esta diferencia funcional. De forma complementaria, las condiciones históricas de uso de suelos y las limitaciones de utilizar una única especie como planta hospedadora expliquen parte de los resultados observados en este estudio. Finalmente, se pone en relieve las limitaciones de los paradigmas actuales de la simbiosis micorrícica y la necesidad de ampliar los estudios en suelos y sistemas complejos analizando las diferentes variables particulares de la comunidad de HMA.
Agradecimientos. Este trabajo fue soportado por PAE-36976-PID53 y PID89 (Agencia Nacional de Promoción Científica y Tecnológica, Argentina) y Secyt-UNC. Agradecemos al IMBIV, al CONICET y la UNC (Argentina) quienes facilitaron los equipos y materiales usados en esta investigación. N.C., C.U., L.W. y A.B. son miembros de la Carrera de Investigador del CONICET. Agradecemos a los Dres. Galantini y Duval (CERZOS-CONICET-UNS-Argentina) por realizar las determinaciones de COT, $\mathrm{P}, \mathrm{NT}$ y pH. También queremos agradecer a los revisores por sus importantes aportes en el presente manuscrito.

\section{REFERENCIAS}

Aguilar Fernández, M., V. J. Jaramillo, L. Varela Fregoso, and M. E. Gavito. 2009. Short term consequences of slashand-burn practices on the arbuscular mycorrhizal fungi of a tropical dry forest. Mycorrhiza 19:179-186.

Alguacil, M. M., E. Lumini, A. Roldán, J. R. Salinas-García, P. Bonfante, and V. Bianciotto. 2008. The impact of tillage practices on arbuscular mycorrhizal fungal diversity in subtropical crops. Ecol Appl 18:527-536.

Bedini, S., L. Avio, C. Sbrana, A. Turrini, P. Migliorini, C. Vazzana, and M. Giovannetti. 2013. Mycorrhizal activity and diversity in a long-term organic Mediterranean agroecosystem. Biol Fertil Soils 49:781-790

Brundrett, M., N. Bougher, B. Dell, T. Grove, and N. Malajczuk. 1996. Working with mycorrhizal in forestry and agriculture. ACIAR Monograph 32.

Burrows, R. 2014. Glomalin Production and Infectivity of Arbuscular-Mycorrhizal Fungi in Response to Grassland Plant Diversity. AM J Plant Sci 5:103-111.

Chagnon, P. L., R. L. Bradley, H. Maherali, and J. N. Klironomos. 2013. A trait-based framework to understand life history of mycorrhizal fungi. Trends Plant Sci 9:484-491.

Clapp, J., J. Young, J. Merryweather, and A. Fitter. 1995. Diversity of fungal symbionts in arbuscular mycorrhizas from a natural community. New Phytol 130:259-265.

Cofré, M. N., A. E. Ferrari, A. Becerra, L. Domínguez, L. G. Wall, and C. Urcelay. 2017. Effects of cropping systems under no-till agriculture on arbuscular mycorrhizal fungi in Argentinean Pampas. Soil Use Manage 33:364-378.

Cuenca G., Z. De Andrade, M. Lovera, L. Fajardo, E. Menenses, M. Marques, and R. Machuca. 2003. Pre-selección de plantas nativas y producción de inóculos de hongos micorrízicos arbusculares (HMA) de relevancia en la rehabilitación de áreas degradadas de la Gran Sabana, estado Bolívar, Venezuela. Ecotropicos (Venezuela) 16:27-40.

Davison, J., M. Öpik, M. Zobel, M. Vasar, M. Metsis, and M. Moora. 2012. Communities of arbuscular mycorrhizal fungi detected in forest soil are spatially heterogeneous but do not vary throughout the growing season. PloS one 7: e41938.

Davison, J., M. Moora, M. Öpik, L. Adholeya, A. Ainsaar, Bâ S. Burla, A. G. Diedhiou, I. Hiiesalu, T. Jairus, N. C. Johnson, A. Kane, K. Koorem, M. Kochar, C. Ndiaye, M. Pärtel, Ü. Reier, Ü. Saks, R. Singh, M. Vasar, and M. Zobel. 2015. Global assessment of arbuscular mycorrhizal fungus diversity reveals very low endemism. Science 28:970973.

Díaz, G., and M. Honrubia. 1993. Infectivity of mine soils from southeast Spain. II. Mycorrhizal population levels in spoilt sites. Mycorrhiza 4:85-88.

Druille, M., M. N. Cabello, P. A. García Parisi, R. A. Golluscio, and M. Omacini. 2015. Glyphosate vulnerability explains changes in root-symbionts propagules viability in pampean grasslands. Agric Ecosyst Environ 202:48-55.

Duval, M. E., J. A. Galantini, J. O. Iglesias, S. Canelo, J. M. Martínez, and L. Wall. 2013. Analysis of organic fractions as indicators of soil quality under natural and cultivated systems. Soil Till Res 131:11-19.

Duval, M. E., J. A. Galantini, J. M. Martínez, F. M. López, and L. G. Wall. 2015. Evaluación de la calidad física de los suelos de la región pampeana: efecto de las prácticas de manejo. Ciencias Agrarias XXV- 15:033-043.

Duponnois, R., C. Plenchette, J. Thioulouse, and P. Cadet. 2001. The mycorrhizal soil infectivity and arbuscular mycorrhizal fungal spore communities in soils of different aged fallows in Senegal. Appl Soil Ecol 17:239-251.

Faggioli, V. S., and M. N. Cabello. 2013. Diversidad de hongos formadores de micorrizas en un gradiente ambiental y de uso de suelo de la provincia de Córdoba Estación Experimental Agropecuaria Marcos Juárez, INTA, 1-6.

Figuerola, E. L. M., L. D. Guerrero, S. M. Rosa, L. Simonetti, M. E. Duval, J. A. Galantini, J. C. Bedano, L. G. Wall, and L. Erijman. 2012. Bacterial Indicator of Agricultural Management for Soil under No-Till Crop Production. PLoS ONE 7: e51075.

Figuerola, E. L. M., L. D. Guerrero, D. Türkowsky, L. G. Wall, and L. Erijman. 2014. Crop monoculture rather than agriculture reduces the spatial turnover of soil bacterial communities at a regional scale. Environ Microbiol DOI: $10.1111 / 1462-2920.12497$. 
Gai, J., W. Gao, L. Liu, Q. Chen, G. Feng, J. Zhang, P. Christie, and X. Li. 2015. Infectivity and community composition of arbuscular mycorrhizal fungi from different soil depths in intensively managed agricultural ecosystems. J Soils Sed 15:1200-1211.

Gange, A. C., and R. L. Ayres. 1999. On the relation between arbuscular mycorrhizal colonization and plant 'benefit'. Oikos 87:615-621.

Ge, Y., J. He, Y. Zhu, J. Zhang, Z. Xu, L. Zhang, and Y. Zheng. 2008. Differences in soil bacterial diversity: driven by contemporary disturbances or historical contingencies? The ISME Journal 2:254-264.

Giovannetti, M., and B. Mosse. 1980. An evaluation of techniques for measuring vesicular arbuscular mycorrhizal infection in roots. New Phytol 84:489-500.

González-Cortés J. C., M. Vega-Fraga, L. Varela-Fregoso, M. Martínez-Trujillo, Y. Carreón-Abud, and M. E. Gavito. 2012. Arbuscular mycorrhizal fungal (AMF) communities and land use change: the conversion of temperate forests to avocado plantations and maize fields in central Mexico. Fungal Ecol 5:16-23.

Grace, C., and D. P. Stribley. 1991. A safer procedure for routine staining of vesicular-arbuscular mycorrhizal fungi. Mycol Res 95:1160-1162.

Grilli, G., C. Urcelay, S. Longo, and L. Galetto. 2014. Mycorrhizal fungi affect plant growth: experimental evidence comparing native and invasive hosts in the context of forest fragmentation. Plant Ecol 215:1513-1525.

Hart, M. M., and R. J. Reader. 2002. Taxonomic basis for variation in the colonization strategy of arbuscular mycorrhizal fungi. New Phytol 153:335-344

Hazard, C., P. Gosling, C. J. van der Gast, D. T. Mitchell, F. N. Doohan, and G. Bending. 2013. The role of local environment and geographical distance in determining community composition of arbuscular mycorrhizal fungi at the landscape scale. The ISME Journal 7:498-508.

Hedlund, K., I. Santa Regina, W. Van der Putten, J. Lepš, T. Díaz, G. Korthals, S. Lavorel, V. Brown, D. Gormsen, and S. Mortimer. 2003. Plant species diversity, plant biomass and responses of the soil community on abandoned land across Europe: idiosyncracy or above belowground time lags. Oikos 103:45-58.

Imaz, P. A., P. A. Barbieri, H. E. Echeverría, H. R. Sainz Rozas, and F. Covacevich. 2014. Indigenous mycorrhizal fungi from Argentina increase Zn nutrition of maize modulated by Zn fertilization. Soil Environm 33(1):23-32.

Irrazabal, G., S. Velázquez, and M. N. Cabello. 2004. Infectividad y diversidad de HMA de la rizosfera de los talares de Magdalena, provincia de Buenos Aires, Argentina. Bol Micol 19:49-57.

Jansa, J., A. Mozafar, G. Kuhn, T. Anken, R. Ruh, I. Sanders, and E. Frossard. 2003. Soil tillage affects the community structure of mycorrhizal fungi in maize roots. Ecol Appl 13:1164-1176.

Jansa, J., A. Wiemken, and E. Frossard. 2006. The effects of agricultural practices on arbuscular mycorrhizal fungi. Geo Soc, London, Special Publications 26:89-115.

Jansa, J., H. R. Oberholzer, and S. Egli. 2009. Environmental determinants of the arbuscular mycorrhizal fungal infectivity of Swiss agricultural soils. Eur J Soil Biol 45:400-408.

Jansa, J., A. Erb, H. R. Oberholzer, P. Šmilauer, and S. Egli. 2014. Soil and geography are more important determinants of indigenous arbuscular mycorrhizal communities than management practices in Swiss agricultural soils. Mol Ecol 23:2118-2135.

Johnson, N. C., P. J. Copeland, R. K. Crookston, and F. Pfleger. 1992. Mycorrhizae: possible explanation for yield decline with continuous corn and soybean. Agronomy Journal 84:387-390.

Johnson, N., J. Graham, and F. Smith. 1997. Functioning of mycorrhizal associations along the mutualism-parasitism continuum. New Phytol 135:575-585.

Johnson, N. C., G. W. Wilson, M. A. Bowker, J. A. Wilson, and R. M. Miller. 2010. Resource limitation is a driver of local adaptation in mycorrhizal symbioses. P Natl Acad Sci USA 107(5):2093-2098.

Karasawa, T., Y. Kasahara, and M. Takebe. 2002. Differences in growth responses of maize to preceding cropping caused by fluctuation in the population of indigenous arbuscular mycorrhizal fungi. Soil Biol Biochem 34:851-857.

Lehmann, A., E. K. Barto, J. R. Powell, M. C. Rillig. 2012. Mycorrhizal responsiveness trends in annual crop plants and their wild relatives - a meta-analysis on studies from 1981 to 2010. Plant Soil 355:231-250.

Longo, S., E. Nouhra, B. T. Goto, R. L. Berbara, and C. Urcelay. 2014. Effects of fire on arbuscular mycorrhizal fungi in the Mountain Chaco Forest. For Ecol Manag 315:86-94.

Lugo, M., and M. N. Cabello. 2002. Native arbuscular mycorrhizal fungi (AMF) from mountain grassland (Córdoba, Argentina) I. Seasonal variation of fungal spore diversity. Mycologia 94:579-586.

Maherali, H., and J. N. Klironomos. 2007. Influence of phylogeny on fungal community assembly and ecosystem functioning. Science 316:1746-1748.

Maherali, H., and J. N. Klironomos. 2012. Phylogenetic and Trait-Based Assembly of Arbuscular Mycorrhizal Fungal Communities. PLoS ONE 7(5): e36695.

Mangan, S. A., A. H. Eom, G. H. Adler, J. B. Yavitt, and E. A. Herre. 2004. Diversity of arbuscular mycorrhizal fungi across a fragmented forest in Panama: insular spore communities differ from mainland communities. Oecologia 141:687-700.

Martínez, T. N., and N. C. Johnson. 2010. Agricultural management influences propagule densities and functioning of arbuscular mycorrhizas in low- and high-input agroecosystems in arid environments. Appl Soil Ecol 46:300-306.

Menéndez, A. B., J. M. Scervino, and A. M. Godeas. 2001. Arbuscular mycorrhizal populations associated with natural and cultivated vegetation on a site of Buenos Aires province, Argentina. Biol Fert Soils 33:373-381. 
Miller, M. H. 2000. Arbuscular mycorrhizae and the phosphorus nutrition of maize: A review of Guelph studies. Can J Plant Sci 80:47-52.

Nadian, H., S. E. Smith, A. M. Alston, and R. S. Murray. 1997. Effects of soil compaction on plant growth, phosphorus uptake and morphological characteristics of vesicular-arbuscular mycorrhizal colonization of Trifolium subterraneum. New Phytol 135:303-311.

Newbold, T., L. N. Hudson, S. L. Hill, S. Contu, I. Lysenko, R. A. Senior, L. Börger, D. J. Bennett, A. Choimes, B. Collen, J. Day, A. De Palma, S. Díaz, S. Echeverría-Londoño, M. J. Edgar, A. Feldman, M. Garon, M. L. Harrison, T. Alhusseini, D. J. Ingram, Y. Itescu, J. Kattge, V. Kemp, L. Kirkpatrick, M. Kleyer, D. L. Correia, C. D. Martin, S. Meiri, M. Novosolov, Y. Pan, H. R. Phillips, D. W. Purves, A. Robinson, J. Simpson, S. L. Tuck, E. Weiher, H. J. White, R. M. Ewers, G. M. Mace, J. P. Scharlemann, and A. Purvis . 2015. Global effects of land use on local terrestrial biodiversity. Nature 520:45-50.

Oehl, F., E. Laczko, A. Bogenrieder, K. Stahr, R. Bösch, M. van der Heijden, and E. Sieverding. 2010. Soil type and land use intensity determine the composition of arbuscular mycorrhizal fungal communities. Soil Biol Biochem 42: 724-738.

Pérez, M., and C. Urcelay. 2009. Differential growth response to arbuscular mycorrhizal fungi and plant density in two wild plants belonging to contrasting functional types. Mycorrhiza 19:517-523.

Plenchette, C., R. Perrin, and P. Duvert. 1989. The concept of soil infectivity and a method for its determination as applied to endomycorrhizas. Can J Bot 67:112-115

Purin, S., O. Klauberg-Filho, and S.L. Sturmer. 2006. Mycorrhizae activity. and diversity in conventional and organic apple orchards from. Brazil. Soil Biol Biochem 38:1831-1839.

Ryan, M. H., and J. H. Graham. 2018. Little evidence that farmers should consider abundance or diversity of arbuscular mycorrhizal fungi when managing crops. New Phytol DOI: 10.1111/nph.15308.

Redecker, D., R. Kodner, and L. E. Graham. 2000. Glomalean fungi from the Ordovician. Science 289:1920-1921.

Renzi, J. P. 2009. Efecto de la estructura de cultivo y grado de madurez a cosecha sobre el rendimiento y la calidad de semillas de Vicia sativa L. y V. villosa Roth., bajo riego. Tesis de Magister en Ciencias Agrarias. Universidad Nacional del Sur. Pp. 126.

Sala, O. E., F. S. Chapin, J. J. Armesto, E. Berlow, J. Bloomfield, R. Dirzo, E. Huber-Sanwald, L. F. Huenneke, R. B. Jackson, and A. Kinzig. 2000. Global biodiversity scenarios for the year 2100. Science 287:1770-1774.

Sanders, I. R. 2004. Plant and arbuscular mycorrhizal fungal diversity- are we looking at the relevant levels of diversity and are we using the right techniques? New Phytol 16:415-418.

Schalamuk, S., S. Velázquez, H. Chidichimo, and M. Cabello. 2006. Fungal spore diversity of arbuscular mycorrhizal fungi associated with spring wheat: effects of tillage. Mycologia 98:16-22.

Schalamuk, S., and M. N. Cabello. 2010a. Effect of Tillage Systems on the Arbuscular Mycorrhizal Fungi (AMF) Propagule Bank in Soils. Pp.162-170 in A. Arya and A. Perelló (eds.). Management of Fugal Plant Pathogens: Current Trends and Progress. Chapter 13. CAB International (publ.).

Schalamuk, S., and M. N. Cabello. 2010b. Arbuscular mycorrhizal fungal propagules from tillage and no-tillage systems: possible effects on Glomeromycota diversity. Mycologia 102:261-268.

Smith, S. E., and D. J. Read. 2008. Mycorrhizal symbiosis. 2nd ed. Academic press Ltd, London.

Soteras, F., D. Renison, and A. G. Becerra. 2014. Restoration of high-altitude forests in an area affected by a wildfire: Polylepis australis Bitt. seedlings performance after soil inoculation. Trees 28(1):173-182

Soteras, F., G. Grilli, N. Cofré, N. Marro, and A. Becerra. 2015. Arbuscular mycorrhizal fungal composition in high montane forests with different disturbance histories in Central Argentina. Appl Soil Ecol 85:30-37.

Stürmer, S. L., and J. O. Siqueira. 2011. Species richness and spore abundance of arbuscular mycorrhizal fungi across distinct land uses in Western Brazilian Amazon. Mycorrhiza 21:255-267.

Treseder, K. K. 2004. A meta-analysis of mycorrhizal responses to nitrogen, phosphorus, and atmospheric CO2 in field studies. New Phytol 164:347-355.

van der Heijden, M. G. A., Bardgett, R. D., and N. M. van Straalen. 2008. The unseen majority: soil microbes as drivers of plant diversity and productivity in terrestrial ecosystems. Ecology Letters 11:296-310.

Vestberg, M., K. Saari, S. Kukkonen, and T. Hurme. 2005. Mycotrophy of crops in rotation and soil amendment with peat influence the abundance and effectiveness of indigenous arbuscular mycorrhizal fungi in field soil. Mycorrhiza 15:447-458.

Wardle, D. A. 2006. The influence of biotic interactions on soil biodiversity. Ecology Letters 9:870-886.

Yang, H. S., Y. Y. Zang, Y. G. Yuan, J. J. Tang, and X. Chen. 2012. Selectivity by host plants affects the distribution of arbuscular mycorrhizal fungi: evidence from ITS rDNA sequence metadata. BMC Evol Biol 12:50. 